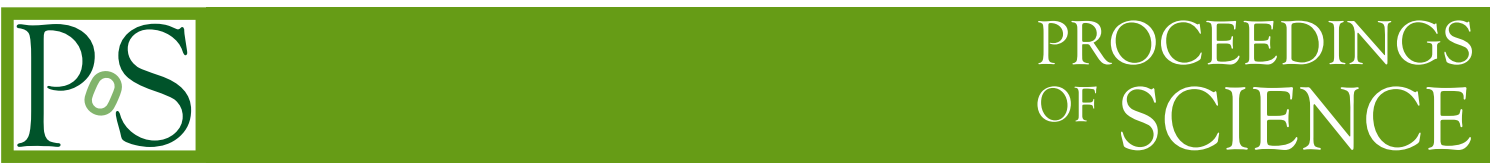

\title{
Scanning the Egress of Vela X-1
}

\author{
P. Kretschmar ${ }^{a}$, I. Kreykenbohm ${ }^{b}$, J. Wilms ${ }^{b}$, M. Hanke ${ }^{b}$, M. Stuhlinger ${ }^{a}$, \\ A.M.T. Pollock ${ }^{a}$, R. Staubert ${ }^{c}$, E. Kendziorra ${ }^{c}$, R. Rothschild ${ }^{d}$ and S. Suchy ${ }^{d}$ \\ E-mail: peter.kretschmardesa.int \\ ${ }^{a}$ ESA - European Space Astronomy Centre, 28691 Villanueva de la Cañada, Madrid, Spain \\ ${ }^{b}$ Dr. Karl Remeis-Sternwarte, 96049 Bamberg, Germany \\ ${ }^{c}$ Institut für Astronomie und Astrophysik, Univ. of Tübingen, 72076 Tübingen, Germany \\ ${ }^{d}$ Center for Astrophysics and Space Science, UCSD, La Jolla, CA, USA
}

\begin{abstract}
We have observed the high mass X-ray binary Vela X-1 shortly after its egress from eclipse on two different occasions: in May 2006 with XMM-Newton and in December 2007 with RossiXTE. Both data sets show a similar behaviour for the earliest observed phases, dominated by the influence of the extended atmosphere of the supergiant, but then marked differences. The XMMNewton observation was characterized by a strong flare and related dramatic changes in the spectral features, while the RossiXTE observations present a much smoother picture
\end{abstract}

7th INTEGRAL Workshop

September 8-112008

Copenhagen, Denmark 


\section{Introduction}

Vela X-1 (4U 0900-40) is an eclipsing high mass X-ray binary (HMXB) consisting of the B0.5Ib super giant HD77581 and a neutron star with an orbital period of 8.964 days [1] at a distance of $\sim 2.0 \mathrm{kpc}$ [2]. The optical companion has a mass of $\sim 23 M_{\odot}$ and a radius of $\sim 30 R_{\odot}$ [1]. Due to the small separation of the binary system with an orbital radius of just $1.7 R_{\star}$, the massive $1.9 M_{\odot}$ neutron star $[3,4]$ is deeply embedded in the dense stellar wind of the optical companion $(\dot{M}=$ $\left.4 \times 10^{-6} M_{\odot} y r^{-1}[5]\right) . X$-ray lines show that this wind is inhomogeneous with many dense clumps [6] being embedded in a much thinner, highly ionized component [7].

Significant random variations in the X-ray pulsar's luminosity - including large flares and sudden drops in luminosity $[8,9,10]$ - indicate rather inhomogenous, clumpy accretion, consistent with this picture.

In order to study the B0Ia supergiant's extended atmosphere and strongly ionized stellar wind we have observed the early part of the orbit on two different occasions with XMM-Newton and RossiXTE (Figure 1).

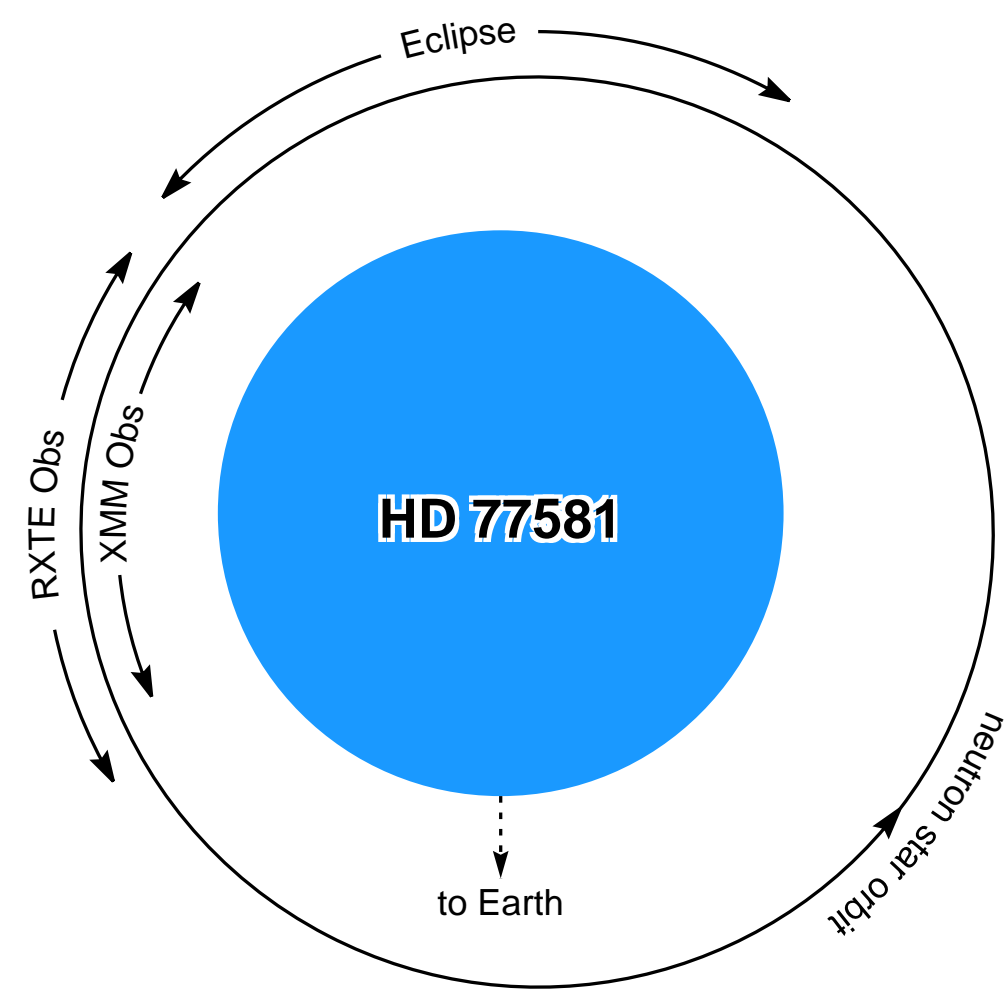

Figure 1: Sketch of the Vela X-1 system indicating the orbital phases covered by the XMM-Newton and RossiXTE observations discussed here. 

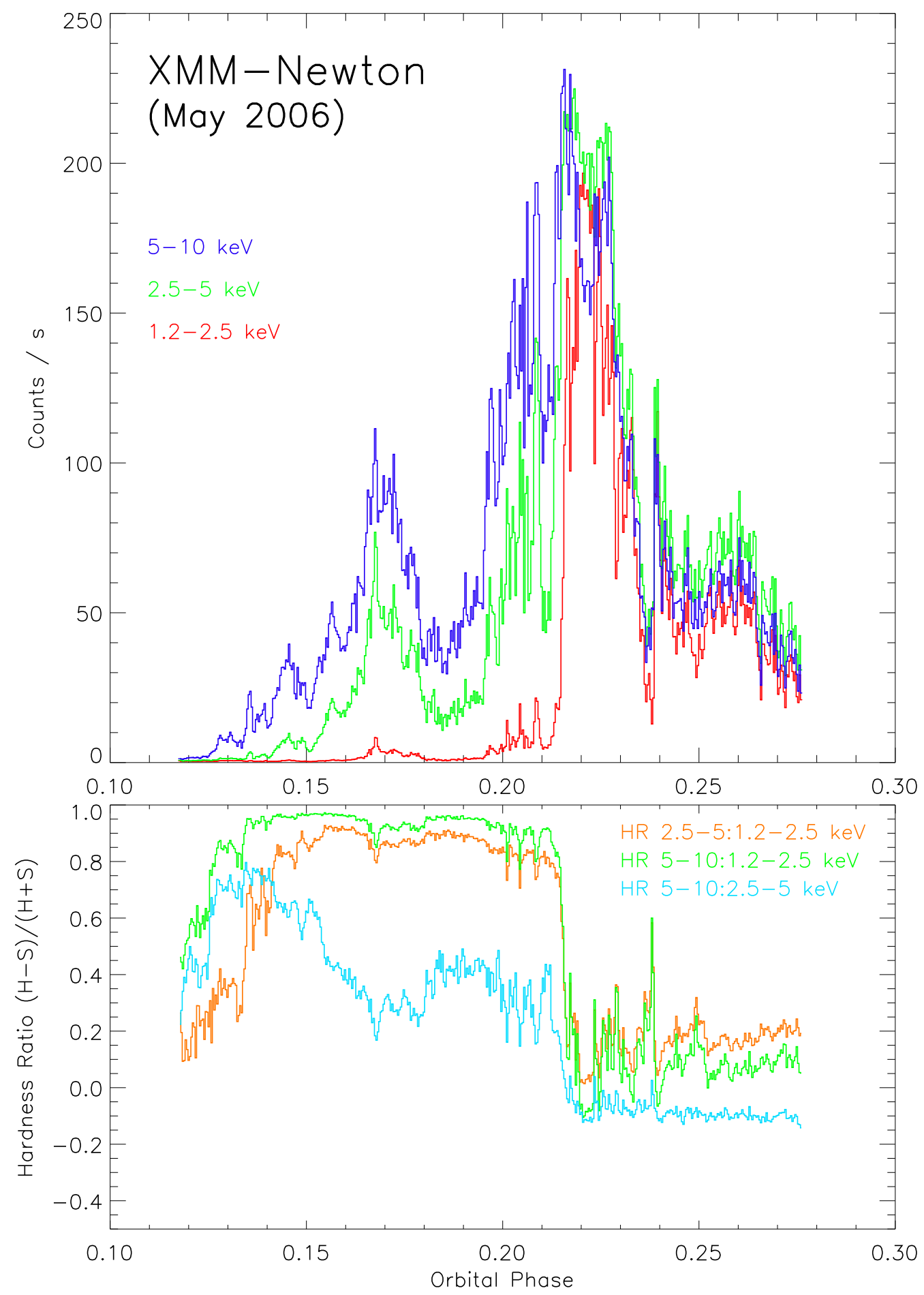

Figure 2: Fluxes in three energy bands and hardness ratios from our XMM-Newton observation in May 2006. At the beginning a hardening is visible related to a slowly diminishing soft excess. During the strong flare after phase 0.2 the spectral hardness changes drastically. 


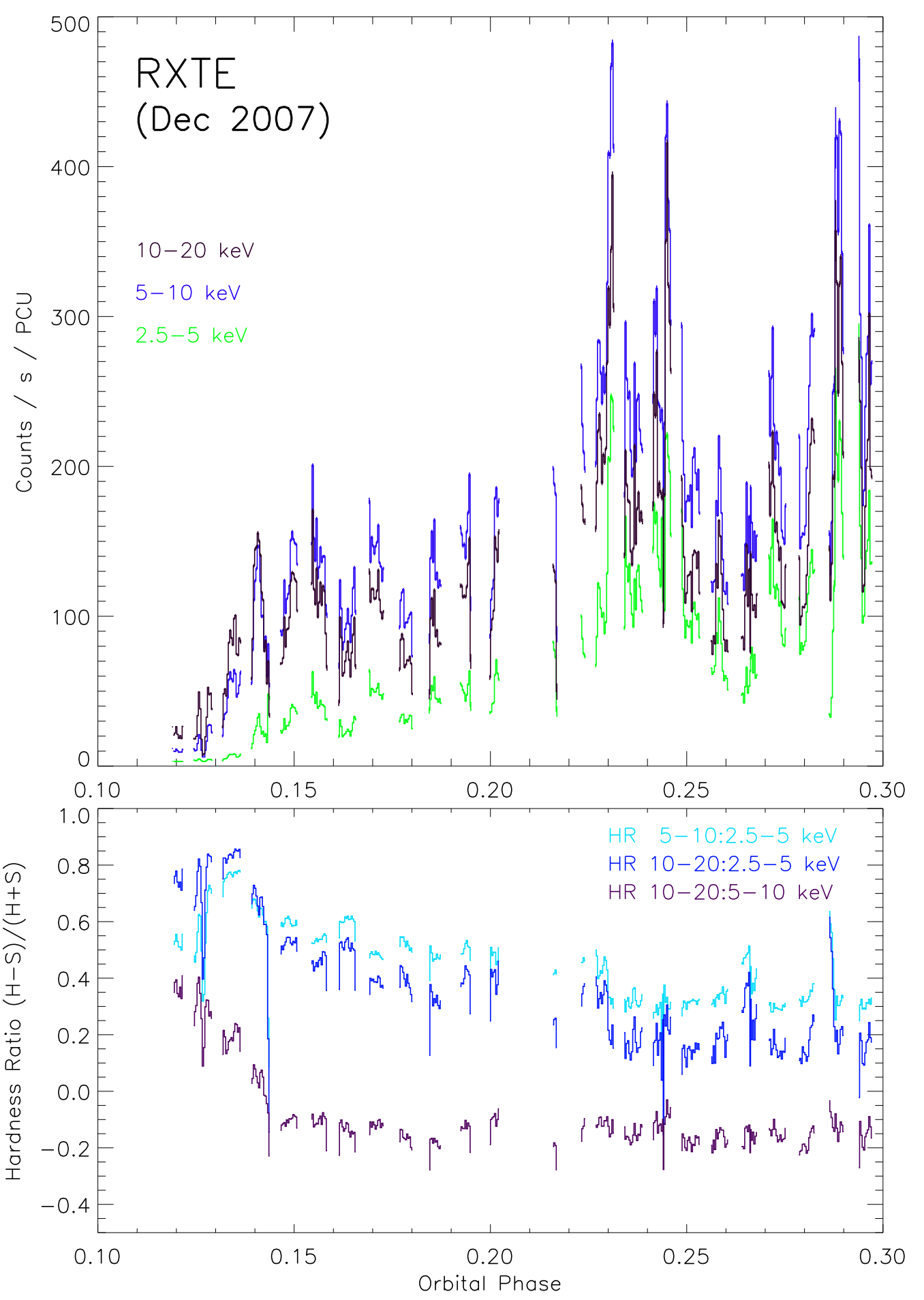

Figure 3: Fluxes in three energy bands and hardness ratios from our RossiXTE observation in Dec 2007. Due to observational constraints the coverage is not continuous and variable in its density. Some flaring activity is also evident in this observation. A soft excess is present before phase 0.14 , after that the spectral hardness evolves relatively smoothly towards a softer spectrum. 


\section{Observations}

The XMM-Newton observation took place during orbital phase $0.12-0.27$ for a total of $123 \mathrm{ks}$ of continuous coverage. The observation was marked by a very dynamic source. During the first half it presented a moderate count rate with smooth variability and several distinct peaks. After phase 0.2 the source flared to such an extent that it saturated the maximum possible telemetry rate of the pn instrument (Figure 2). Going through a narrow minimum at orbital phase $\sim 0.24$ the source returned to roughly its pre-flare level.

With RossiXTE a sequence of observations of varying length was scheduled spanning orbital phases $0.05-0.30$ for a total of $105 \mathrm{ks}$. In this preliminary analysis we only used observations with a clearly visible source, covering phases $0.12-0.30$ (Figure 3 ). The source evolution was less dramatic than during the earlier XMM-Newton observation, but again some flaring activity is visible after orbital phase $\sim 0.23$.

\section{Results}

For the whole XMM-Newton observation, the spectra above $\sim 2 \mathrm{keV}$ are well described by an absorbed power law component plus Fe-line emission. In addition, a soft excess was present at lower energies especially for the earlier orbital phases. Before the major flare, several emission line features of $\mathrm{Si}, \mathrm{Mg}$ and $\mathrm{Ne}$ were detected.

Besides the overall countrate, the XMM-Newton observations also presented a peculiar and dramatic evolution of the absorption column density $N_{\mathrm{H}}$. After an initial fall-off it remained roughly constant for $\sim$ half a day and then dropped rapidly by roughly one order of magnitude (see Figure 5).

Also the absolute and relative strength of the Fe-K fluorescence line varied significantly over this observation (see Figure 4). Around orbital phase 0.18, coinciding with a drop in overall flux and a rise in $N_{\mathrm{H}}$, it increased by a factor $\sim 3$. Between phases 0.21 and 0.22 the relative line intensity dropped sharply to the previous level, at about the same time as the overall flux peaked and $N_{\mathrm{H}}$ dropped to its minimum.

In contrast to these dramatic changes, the RossiXTE observations show a much smoother evolution. The spectra are well described in the range $3-20 \mathrm{keV}$ by an absorbed power law with a cutoff towards higher energies plus Fe-line emission. The well known cyclotron line features with a harmonic at $\sim 25 \mathrm{keV}$ are visible, but not the topic of this work. Again a soft excess is visible in the early phases, though less clearly than with XMM-Newton, due to the energy range covered.

Spectral hardness (Figure 3) and $N_{\mathrm{H}}$ (Figure 5) show a much smoother evolution than during the earlier observation, with a small rapid change at orbital phase 0.23 , coincident with the onset of flaring. 


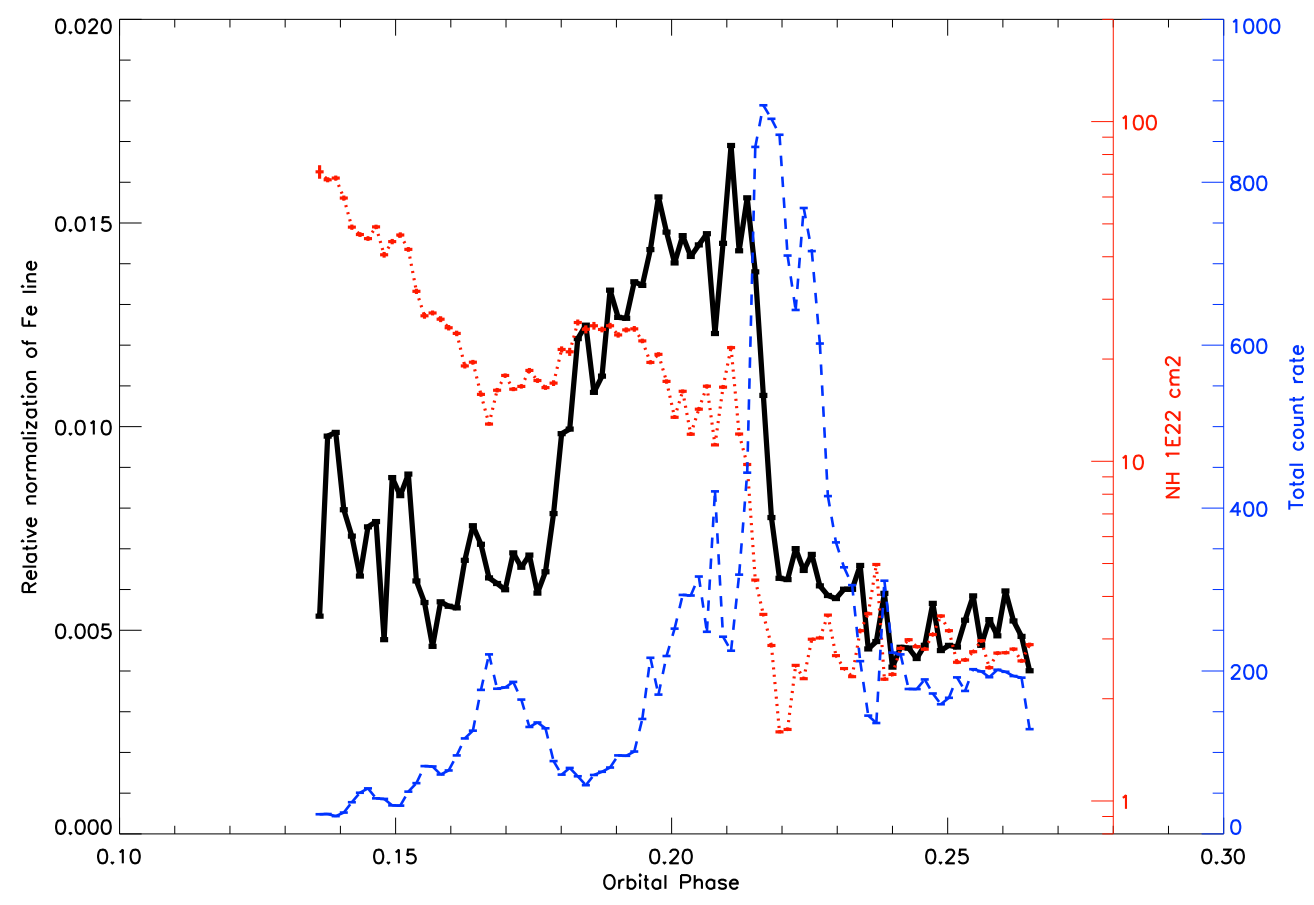

Figure 4: Relative strength of the Fe-K line over orbital phase, based on the XMM-Newton observation. For comparison, the evolution of $N_{\mathrm{H}}$ and the best fit normalization are also shown.

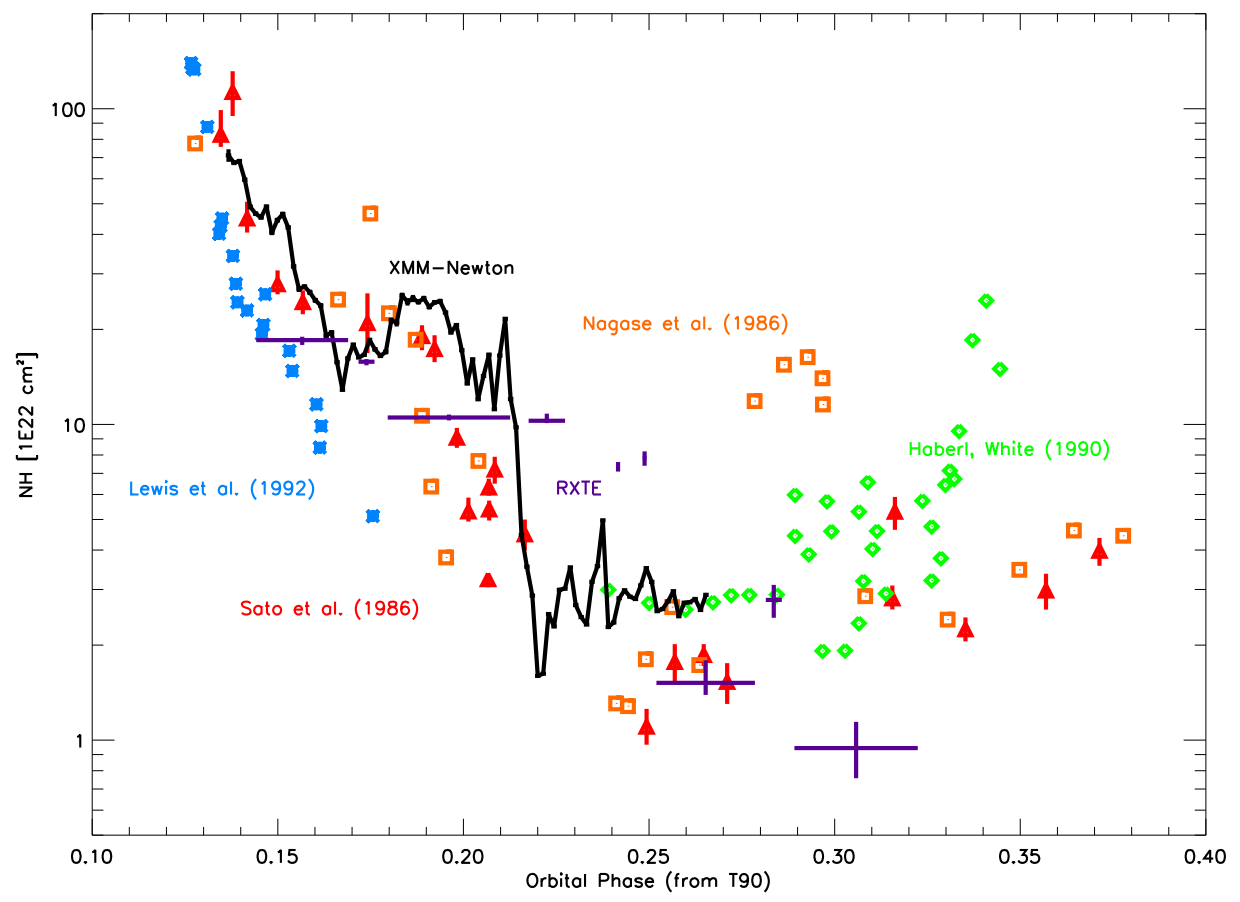

Figure 5: Comparison of historical measurements of $N_{\mathrm{H}}$ with the values obtained from our observations. The historical data have been corrected for differences in zero phase definition as far as possible. 


\section{Discussion}

The comparison of these two detailed observations demonstrates again the well-known large variability in the behaviour of Vela X-1 [9], but also some common features, e.g., the early steep fall in $N_{\mathrm{H}}$ and the soft excess.

A comparison with historical measurements (Figure 5) underlines the variability and sometimes rapid $N_{\mathrm{H}}$ variations, with a tempting indication of a trend to increased $N_{\mathrm{H}}$ values around phase 0.2 , which would need confirmation.

The dramatic flare and associated spectral evolution observed with XMM-Newton might be understood by the capture of a dense cloud of material which first enshrouds the X-ray source and scatters its light and then gets rapidly accreted fueling the main flare.

Work is ongoing for a more detailed analysis of the spectral evolution in both data sets, preparing the ground for a comparison with the predictions of hydrodynamical simulation models [11].

\section{References}

[1] M. H. van Kerkwijk, J. van Paradijs, E. J. Zuiderwijk, G. Hammerschlag-Hensberge, L. Kaper, and C. Sterken, Spectroscopy of HD 77581 and the mass of Vela X-1, A\&A 303 (1995) 483.

[2] F. Nagase, Accretion powered X-ray pulsars, PASJ 41 (1989) 1-79.

[3] H. Quaintrell, A. J. Norton, T. D. C. Ash, P. Roche, B. Willems, T. R. Bedding, I. K. Baldry, and R. P. Fender, The mass of the neutron star in Vela X-1 and tidally induced non-radial oscillations in GP Vel, A\&A 401 (Apr., 2003) 313-323.

[4] O. Barziv, L. Kaper, M. H. Van Kerkwijk, J. H. Telting, and J. Van Paradijs, The mass of the neutron star in Vela X-1, A\&A 377 (Oct., 2001) 925-944.

[5] F. Nagase, S. Hayakawa, and N. Sato, Circumstellar matter in the Vela X-1/HD 77581 system, PASJ 38 (1986) 547-569.

[6] L. M. Oskinova, W.-R. Hamann, and A. Feldmeier, X-raying clumped stellar winds, in Clumping in Hot-Star Winds (W.-R. Hamann, A. Feldmeier, and L. M. Oskinova, eds.), 203, 2008.

[7] M. Sako, D. A. Liedahl, S. M. Kahn, and F. Paerels, The X-Ray Spectrum and Global Structure of the Stellar Wind in VELA X-1, ApJ 525 (Nov., 1999) 921-934.

[8] R. Krivonos, N. Produit, I. Kreykenbohm, R. Staubert, A. von Kienlin, C. Winkler, and N. Gehrels, $A$ very intense X-ray outburst of Vela X-1 detected with INTEGRAL, The Astronomer's Telegram 211 (Dec., 2003).

[9] I. Kreykenbohm, J. Wilms, P. Kretschmar, J. Torrejón, K. Pottschmidt, M. Hanke, A. Santangelo, C. Ferrigno, and R. Staubert, High variability in Vela X-1: giant flares and off states, $A \& A$ accepted (July, 2008).

[10] P. Kretschmar, I. Kreykenbohm, J. Wilms, R. Staubert, W. Heindl, D. Gruber, and R. Rothschild, Disappering pulses in Vela X-1, in Proceedings of the Fifth Compton Symposium (M. L. McConnell and J. M. Ryan, eds.), no. 510 in CP, (Woodbury, New York), pp. 163-167, AIP, 2000.

[11] C. W. Mauche, D. A. Liedahl, S. Akiyama, and T. Plewa, Hydrodynamic and Spectral Simulations of HMXB Winds, Progress of Theoretical Physics Supplement 169 (2007) 196-199, [arXiv:0704.0237]; 